\title{
An Innovational, Collision Model and Data Set Generation at Novel Test-Rig for Validation of Numerical Model in the Frame of Machine Learning
}

\author{
Agata Widuch $^{1}$, Marcin Nowak ${ }^{1}$, Ziemowit Ostrowski ${ }^{1}$, Adam Klimanek ${ }^{1}$, Ryszard Białecki ${ }^{1}$, Kari \\ Myöhänen ${ }^{2}$, Alessandro Parente ${ }^{3}$ and Wojciech Adamczyk ${ }^{1}$ \\ ${ }^{1}$ Silesian University of Technology, Department of Thermal Technology, 44-100 Gliwice, Konarskiego 22, Poland, \\ agata.widuch@polsl.p1* \\ ${ }^{2}$ LUT School of Energy Systems, Lappeenranta University of Technology, Post Office Box 20, FI-53851 Lappeenranta, \\ Finland \\ ${ }^{3}$ Aero-Thermo-Mechanics Laboratory, Ecole polytechnique de Bruxelles, Université Libre de Bruxelles, Avenue Franklin \\ D. Roosevelt 50, CP 165/41, Brussels 1050, Belgium
}

\section{Extended Abstract}

Granular flows are characterised by the high volume fraction of solid phase. To overcome the necessity of building real scale installations required to improve process where particulate transport is involved there is an urgent need of building a mathematical models capable to accurately simulate processes involving great number of particles. While simulating granular flows, the modelling mutual particle interactions still remains the greatest and most time consuming challenge. Due to complexity and great number of interactions, modeling such flows is not a trivial task. Nowadays two main models are available hybrid Euler-Lagrange (HEL) [1] and discrete element method (DEM) [2]. In HEL, interactions between solid phase are determined on the basis of Kinetic Theory of Granular Flow (KTGF), where probability of collision is determined from solid volume fraction in computational cell but time needed to obtain results is relatively short. In DEM, calculating every possible particles interactions is computationally expensive as well as time consuming. To overcome the problem of long lasting calculations and predicting particle interactions, while keeping required accuracy a fast and robust Reduced Model developed based on the algorithms of machine learning. The principle of the idea is to use the large set of particle data (before and after collision) and on their basis to build machine learning model capable to predict the new values of ex. velocities.

In such a way created model will be used as a part of HEL approach to substitute forces between particle interactions basing on solid volume fraction with forces obtained using DEM. In such a way the precision of DEM model will be preserved but results will be obtained much faster. Parallel to creating the Reduced Model the experiment tests were carried out. The collected data will serve as the validation set for further analysis. Both experimental and simulation results will be compared using computer vision algorithms.

Keywords: Granular Flows, Multiphysics Problems, Computing Methods, Reduced Models, Computer Vision.

This research is supported by National Science Centre Poland within projects No UMO-2018/31/B/ST8/02201. This help is gratefully acknowledged herewith.

\section{References}

[1] D. Gidaspow, V. Jiradilok, Computational techniques, the multiphase CFD approach to fluidization and green energy technologies, 1st Edition, Nova Science Publishers, Inc., 2009.

[2] C. Crowe, J. Schwarzkopf, M. Sommerfeld, Y. Tsuji, Multiphase flows with droplets and particles, Taylor \& Francis group, 2012. 